\title{
Does Increased Regularity Lower Complexity?
}

\author{
By Arthur G. Werschulz*
}

\begin{abstract}
Intuitively, the more regular a problem, the easier it should be to solve. Examples drawn from ordinary and partial differential equations, as well as from approximation, support the intuition. Traub and Wozniakowski conjectured that this is always the case. In this paper, we study linear problems. We prove a weak form of the conjecture, and show that this weak form cannot be strengthened. To do this, we consider what happens to the optimal error when regularity is increased. If regularity is measured by a Sobolev norm, increasing the regularity improves the optimal error, which allows us to establish the conjecture in the normed case. On the other hand, if regularity is measured by a Sobolev seminorm, it is no longer true that increasing the regularity improves the optimal error. However, a "shifted" version of this statement holds, which enables us to establish the conjecture in the seminormed case.
\end{abstract}

1. Introduction. We investigate the relation between regularity and complexity. In this Introduction, we use words such as algorithm, information, cardinality, and regularity without definition. They are rigorously defined later.

Based on a variety of examples, Traub and Woźniakowski [6] conjectured that, in general, as the regularity of a class of problem elements increases, the complexity decreases. In this paper, we consider linear problems. We measure regularity by a Sobolev norm or seminorm. We prove a weak form of this conjecture and show that no stronger statement is possible.

To fix ideas, we consider several examples.

Example 1.1. Consider the solution of the two-point boundary-value problem

$$
-u^{\prime \prime}=f \quad \text { in }(0,1), \quad u(0)=u(1)=0,
$$

where the $H^{r}(0,1)$-norm of $f$ is bounded by unity:

$$
\sum_{j=0}^{r} \int_{0}^{1}\left|f^{(j)}(x)\right|^{2} d x \leqslant 1 .
$$

Consider an algorithm $\varphi$ using information of cardinality at most $n$, and define the error $e(\varphi)$ to be the worst-case error (in the $H^{1}$-sense) taken over all $f$ satisfying (1.2). Let

$$
e(n, r):=\inf _{\varphi} e(\varphi)
$$

Received October 21, 1981; revised January 27, 1983.

1980 Mathematics Subject Classification. Primary 65N15, 65N30, 68C25; Secondary 47A70, 47B05, $65 \mathrm{~J} 10$.

*This research was supported in part by the National Science Foundation under Grant MCS-8203271. 
be the minimal error of all such m-evaluation algorithms $\varphi$ whose input functions $f$ satisfy (1.2). In [7], we showed that

$$
e(n, r)=\Theta\left(n^{(r+1)}\right) \text { as } n \rightarrow \infty .
$$

where we use Knuth's $\Theta$-notation

$$
f_{1}=\Theta\left(f_{2}\right) \text { iff } f_{1}=O\left(f_{2}\right) \text { and } f_{2}=O\left(f_{1}\right) .
$$

If $\operatorname{comp}(\varepsilon, r)$ denotes the complexity of finding an $\varepsilon$-approximation, then (1.4) implies

$$
\operatorname{comp}(\varepsilon, r)=\Theta\left(\left(\frac{1}{\varepsilon}\right)^{1 / 1 r+1)}\right) \quad \text { as } \varepsilon \rightarrow 0
$$

The next four examples are taken from [6]. In these examples, the data consisted of all $f \in H^{r}(I)$ (where $I$ was a bounded real interval) whose $H^{r}(I)$-seminorm was bounded by unity:

$$
\int_{I}\left|f^{(r)}(x)\right|^{2} d x \leqslant 1
$$

For an algorithm $\varphi$ using information of cardinality at most $n, e(\varphi)$ was defined to be the $L_{2}$-error taken over all $f$ satisfying (1.7), and

$$
e(n, r):=\inf _{\varphi} e(\varphi)
$$

was the minimal error of all such $n$-evaluation algorithms $\varphi$ whose input functions $f$ satisfy (1.7). Once again, $\operatorname{comp}(\varepsilon, r)$ denotes the complexity of finding an $\varepsilon$-approximation.

Example 1.2. For the approximation problem,

$$
e(n, r)=\Theta\left(n^{-r}\right) \text { as } n \rightarrow \infty .
$$

so that

$$
\operatorname{comp}(\varepsilon, r)=\Theta\left(\left(\frac{1}{\varepsilon}\right)^{1 / r}\right) \text { as } \varepsilon \rightarrow 0
$$

Example 1.3. For the heat equation in a thin rod of length $\pi$ with initial data $f$ solved out to time $t=t_{0}$,

$$
e(n, r)=e^{-(n+1)^{2} t_{0}}(n+1)^{-r},
$$

so that

$$
\operatorname{comp}(\varepsilon, r)=\Theta\left(\sqrt{\frac{1}{t_{0}} \ln \frac{1}{\varepsilon}}\right) \quad \text { as } \varepsilon \rightarrow 0
$$

Example 1.4. For the Laplace equation on the square $(0, \pi) \times(0, \pi)$ with boundary data

$$
\begin{cases}u(0, y)=u(\pi, y)=u(x, 0)=0 & \text { for } x, y \in[0, \pi], \\ u(x, \pi)=f(x) & \text { for } x \in[0, \pi],\end{cases}
$$

and considering the solution to be $u\left(\cdot, y_{0}\right)$ for a fixed $y_{0} \in(0, \pi)$,

$$
e(n, r)=\frac{1}{(n+1)^{r}} \frac{\sinh (n+1) y_{0}}{\sinh (n+1) \pi} \sim e^{-(n+1)\left(n-y_{0}\right)}(n+1)^{-r} \text { as } n \rightarrow \infty,
$$


so that

$$
\operatorname{comp}(\varepsilon, r)=\Theta\left(\frac{1}{\pi-y_{0}} \ln \frac{1}{\varepsilon}\right) \quad \text { as } \varepsilon \rightarrow 0 .
$$

Example 1.5. For the hyperbolic differential equation

$$
\left\{\begin{array}{l}
\frac{\partial u}{\partial t}=\frac{\partial u}{\partial x} \quad(x \in \mathbf{R}, t>0) \\
u(\cdot, 0)=f
\end{array}\right.
$$

solved out to time $t=t_{0}$,

$$
e(n, r)=(n+1)^{-r}
$$

so that

$$
\operatorname{comp}(\varepsilon, r)=\Theta\left(\varepsilon^{-1 / r}\right) \quad \text { as } \varepsilon \rightarrow 0 .
$$

(See Chapter 6 of [6] for a fuller discussion.)

Note that, in all the examples above, $s>r$ implies that

$$
\lim _{n \rightarrow \infty} \frac{e(n, s)}{e(n, r)}=0
$$

and that there is a constant $K$ which is close to unity such that

$$
\limsup _{\varepsilon \rightarrow 0} \frac{\operatorname{comp}(\varepsilon, s)}{\operatorname{comp}(\varepsilon, r)} \leqslant K .
$$

Hence, as the regularity increases, the complexity decreases, in the sense that it gets no worse. Traub and Woźniakowski [6, p. 147] asked whether more regular problems always have lower complexity. We add the question as to whether (1.19) holds in general.

In order to establish the conjecture of [6], it is necessary to first determine what happens to the $n$th minimal error $e(n, r)$ as $r$ is increased. Let $s>r$. We show that for any problem, there exist nonnegative integers $n_{r}^{*}$ and $n_{s}^{*}$, with $n_{r}^{*} \leqslant n_{s}^{*}$, such that

$$
\lim _{n \rightarrow \infty} \frac{e\left(n+n_{s}^{*}, s\right)}{e\left(n+n_{r}^{*}, r\right)}=0
$$

in both the normed and seminormed cases. In this sense, additional regularity always helps. However, (1.20) tells us nothing about the more fundamental question of whether (1.19) holds. We now distinguish between the normed and seminormed cases. In the normed case, $n_{r}^{*}=n_{s}^{*}=0$, so that (1.19) holds; we also have the nonasymptotic result that

$$
e(n, s) \leqslant e(n, r) \text { for } n \geqslant 0 .
$$

In the seminormed case, (1.20) implies the desired result (1.19) when the problem is "hard", and so the "shift" is irrelevant. In general, however, we cannot say that (1.20) implies (1.19); in fact, we are able to construct a special counterexample in the seminormed case for which

$$
\lim _{n \rightarrow \infty} \frac{e(n, s)}{e(n, r)}=\infty
$$


(and the limit can blow up arbitrarily fast). Note that this counterexample is an "easy" problem, especially constructed for this purpose; we know of no naturallyoccurring problem for which (1.22) holds.

We are now able to use (1.20) and (1.21) to establish a weak form of the original conjecture: in both the normed and seminormed cases, there is a constant $K_{1}$ close to unity such that

$$
\limsup _{\varepsilon \rightarrow 0} \frac{\operatorname{comp}(\varepsilon, s)}{\operatorname{comp}(\varepsilon, r)} \leqslant K_{1} .
$$

On the other hand, this is essentially the sharpest statement possible: in both the normed and seminormed cases, one can always construct a problem for which

$$
\limsup _{\varepsilon \rightarrow 0} \frac{\operatorname{comp}(\varepsilon, s)}{\operatorname{comp}(\varepsilon, r)} \geqslant K_{2},
$$

where $K_{2}$ is close to unity. In other words, increasing regularity improves complexity, but not as dramatically as the optimal error is improved in the normed case; it is not true in general that

$$
\lim _{\varepsilon \rightarrow 0} \frac{\operatorname{comp}(\varepsilon, s)}{\operatorname{comp}(\varepsilon, r)}=0 .
$$

We now outline the contents of this paper. In Section 2, we develop our terminology and introduce some known results on optimal algorithms. In Section 3 , we prove a useful theorem on ratios of eigenvalues. In Section 4, this theorem is applied to the normed case to give results on optimal error. We discuss optimal error in the seminormed case in Section 5. The results in Sections 4 and 5 are translated into results on complexity in Section 6, where we establish the weak form of the conjecture and show that no stronger version is possible. Finally, we pose some open questions in Section 7.

2. Preliminary Concepts. In this section, we introduce some terminology from [6], which will more precisely define some of the terms mentioned in the Introduction. We also mention some results from [6] concerning optimal algorithms.

Let $\widetilde{F}_{1}, \widetilde{F}_{2}$ be (real or complex) Hilbert spaces. A problem is defined by a bounded linear solution operator $S: \bar{y}_{0} \rightarrow \bar{y}_{2}$, where $\bar{F}_{0} \subset \bar{y}_{1}$ is a set of problem elements. For our purposes, we may assume that there is a surjective restriction operator $T$ : $\widetilde{F}_{1} \rightarrow \widetilde{F}_{3}\left(\widetilde{F}_{3}\right.$ a Hilbert space $)$ such that

$$
{\widetilde{F_{0}}}_{0}=\left\{f \in \bar{F}_{1}:\|T f\| \leqslant 1\right\} .
$$

For instance, if $\mathscr{F}_{3}=\widetilde{F}_{1}$ and $T=I$ (the identity map), $\widetilde{\mathscr{F}}_{0}$ becomes the unit ball $B \widetilde{F_{1}}$ of $\mathscr{F}_{1}$. (In the sequel, $B H$ will denote the unit ball of any Hilbert space $H$.)

In what follows, we let $\Omega \subset \mathbf{R}^{p}$ be a smooth bounded domain. We use the standard notation and terminology for multi-indices, as well as Sobolev norms, seminorms, inner products, and spaces; see, e.g., [2].

Remark 2.1. Let $\mathscr{F}_{3}=\mathscr{F}_{1}=H^{r}(\Omega)$ and $T=I$, so that

$$
\mathscr{F}_{0}=B H^{r}(\Omega)=\left\{f \in H^{r}(\Omega):\|f\|_{r} \leqslant 1\right\} .
$$

This is the setting for the normed case as discussed in the Introduction. On the other hand, choose $\mathscr{F}_{1}=H^{r}(\Omega), m$ to be the number of $p$-dimensional multi-indices of 
order $r$, and $\bar{F}_{3}$ to be the (closed) subspace of $L_{2}(\Omega)^{m}$ which is the range of the transformation $T$ which maps a function in $H^{r}(\Omega)$ to the vector of its partial derivatives of order $r$. We then find that

$$
\widetilde{\mathscr{F}}_{0}=\mathscr{B}^{r} H^{r}(\Omega):=\left\{f \in H^{r}(\Omega):|f|_{r} \leqslant 1\right\} .
$$

This is the setting for the seminormed case. (See Section 6.)

In order to clarify our terminology, we now introduce

Example 2.1. Choose $\mathscr{F}_{1}=H^{r}(\Omega)$ where $r \geqslant-1, \mathscr{F}_{2}=H_{0}^{1}(\Omega)$, and $\mathscr{F}_{0}$ to be the unit ball of $H^{r}(\Omega)$. The solution operator $S: \mathscr{F}_{0} \rightarrow \mathscr{F}_{2}$ is defined by letting $S f$ be the solution to

$$
\int_{\Omega} \nabla(S f) \cdot \nabla v=\int_{\Omega} f v \quad \forall v \in H_{0}^{1}(\Omega),
$$

i.e., $u=S f$ is the weak solution to

$$
\begin{aligned}
& -\Delta u=f \quad \text { in } \Omega \\
& u=0 \text { on } \partial \Omega \text {, }
\end{aligned}
$$

see e.g. [2].

In order to approximate the solution $S f$ for $f \in \mathscr{F}_{0}$ using a finite amount of resources, we must use only a finite amount of information. Here, an information operator is a linear operator $\mathfrak{K}: D_{\Re} \rightarrow \mathscr{F}_{4}$ (where $\mathscr{F}_{0} \subset D_{\Re} \subset \mathscr{F}_{1}$ and $\mathscr{F}_{4}$ is a Hilbert space) whose cardinality $\# \mathcal{X}$ is given by

$$
\# \Re:=\text { codimker } \Re \text {. }
$$

From Chapter 2 of [6], \# $\Re=n$ if and only if there exist $n$ linearly independent linear functionals $L_{1}, \ldots, L_{n}$ on $\mathscr{F}_{1}$ such that

$$
\Re f=\left[L_{1} f \cdots L_{n} f\right]^{T} \quad \forall f \in \mathscr{F}_{0} .
$$

Example 2.1 (continued). One important information operator for this problem is given by

$$
\tilde{\mathscr{T}}_{n} f:=\left[\int_{\Omega} f s_{1}^{n} \cdots \int_{\Omega} f s_{n}^{n}\right]^{T}
$$

where $s_{1}^{n}, \ldots, s_{n}^{n}$ form a basis for a space $\delta_{n} \subset H_{0}^{1}(\Omega)$ of piecewise polynomials of degree $r$, and the sequence $\left\{\delta_{n}\right\}_{n \geqslant 1}$ arises from a quasi-uniform triangulation of $\Omega$. (Of course, if $p \geqslant 2$, we must make some adjustments to guarantee that $\delta_{n} \subseteq H_{0}^{1}(\Omega)$. For more details, see [2] and [7].)

An algorithm using $\Re$ is then a (not-necessarily-linear) mapping $\varphi: \mathcal{T}\left(\mathscr{F}_{0}\right) \rightarrow \mathscr{F}_{2}$. (Hence the only information such an algorithm may use about the problem element $f \in \mathscr{F}_{0}$ is $\Re f$.) The (worst-case) error $e(\varphi)$ of such an algorithm $\varphi$ using $\Re$ is then defined by

$$
e(\varphi):=\sup _{f \in \mathscr{T}_{0}}\|S f-\varphi(\Re f)\| .
$$

Example 2.1 (continued). Define $\tilde{\varphi}_{n}$ to be the finite element algorithm, i.e., $\tilde{\varphi}_{n}\left(\tilde{\mathscr{T}}_{n} f\right) \in \delta_{n}$ satisfies

$$
\int_{\Omega} \nabla \tilde{\varphi}_{n}\left(\tilde{\mathscr{T}}_{n} f\right) \cdot \nabla s_{i}^{n}=\int_{\Omega} f s_{i}^{n} \quad(1 \leqslant i \leqslant n) .
$$


Then $\tilde{\varphi}_{n}$ uses $\mu_{n}$ and

$$
e(\tilde{\varphi})=A\left(n^{-1,+11 / p}\right) \text { as } n \rightarrow \infty \text {. }
$$

(See $[2],[7] ;=$

We seth optimal error algorithms $q^{*}$ using whose error is small as possible, i.e., such that

$$
e\left(\varphi^{*}\right)=\inf _{\varphi} e(\varphi),
$$

the infimum being over all $\varphi$ using $\pi$. The search is made easier by the fact that

$$
\inf _{\varphi} e(\varphi)=r\left(x, S, \overline{v_{r}}\right) \text {. }
$$

where the radius of information is given by

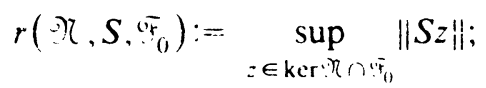

see Chapters 1 and 2 of [6].

Example 2.1 (continued). The finite element algorithm $\tilde{\varphi}_{n}$ is (to within a constant factor independent of $n$ ) of optimal error among all algorithms using $\bar{x}_{n}$; see [7].

Now that we are able to determine the optimal error for any algorithm using information of cardinality at most $n$, the next matter to determine is which such information is "most relevant" in that it yields optimal algorithms with the smallest error. That is, we wish to find an $n$th optimal information operator $\mathfrak{x}_{n}$, i.e., an information operator $\varkappa_{n}$ of cardinality at most $n$ whose radius equals the $n$th minimal radius of information:

$$
r\left(\Re_{n}, S, \bar{y}_{0}\right)=r\left(n, S, \bar{r}_{0}\right):=\inf _{\# \leqslant n} r\left(\mathcal{N}, S, \bar{v}_{0}\right) .
$$

We will mainly be concerned with the case where

$$
\lim _{n \rightarrow \infty} r\left(n, S, \bar{v}_{0}\right)=0 \text {, }
$$

so that there exists a convergent sequence of algorithms, each of which uses information of finite cardinality. By Corollary 2.5.1 of [6], (2.16) holds if and only if $K:=S T^{\dagger}$ is compact, $T^{\dagger}$ being a pseudo-inverse of $T$ (see p. 34 of [6]). Let $\lambda_{1} \geqslant \lambda_{2} \geqslant \cdots>0$ be the nonzero eigenvalues of $K^{*} K$, the asterisk denoting Hilbert space adjoint. (If only a finite number $r$ of nonzero eigenvalues, formally set $\lambda_{1}=0$ for $i>r$.) Let

$$
n^{*}=n^{*}(T, S):=\operatorname{dim}[\operatorname{ker} T /(\operatorname{ker} T \cap \operatorname{ker} S)]
$$

denote the problem index. Then Theorems 2.3.2 and 2.5.3 of [6] yield

$$
r\left(n, S, \widetilde{\mathscr{F}}_{0}\right)= \begin{cases}\infty & \text { if } n<n^{*}, \\ \sqrt{\lambda_{n-n^{*}+1}} & \text { if } n \geqslant n^{*} .\end{cases}
$$

Remark 2.2. In the normed setting $\widetilde{F}_{0}=B H^{r}(\Omega)$, we have $T=I$, so that $T^{\dagger}=I$, and hence $n^{*}=0$. In the seminormed setting $\widetilde{F}_{0}=G_{B} H^{r}(\Omega)$, we will show that $n^{*}=\operatorname{dim}\left(S\left(P_{r-1}(\Omega)\right)\right)$, where $P_{t}(\Omega)$ is the space of polynomials of degree $t$ over the region $\Omega$.

Example 2.1 (continued). In [6], we showed that

$$
r\left(\tilde{\mathcal{T}}_{n}, S, \mathscr{F}_{0}\right)=\Theta\left(r\left(n, S, \widetilde{F}_{0}\right)\right)=\Theta\left(n^{-(r+1) / p}\right) \quad \text { as } n \rightarrow \infty .
$$


Hence, $\tilde{\mathfrak{X}}_{n}$ is (to within a constant factor, independent of $n$ ) an $n$th optimal information for the problem (2.4).

3. An Eigenvalue Comparison Theorem. In the previous section, we saw how the minimal radii of information were related to the eigenvalues of a compact linear operator on a Hilbert space. In this section, we will establish a result concerning eigenvalues of products of compact linear transformations. This result will be used to establish results on optimal error for the normed and seminormed cases in Sections 4 and 5 , respectively.

Let $X$ and $Y$ be Hilbert spaces, and let $E: X \rightarrow Y$ and $A: Y \rightarrow Y$ be compact linear transformations, with $A$ selfadjoint and nonnegative, i.e.

$$
A=A^{*} \text { and }(A y, y) \geqslant 0 \text { for } y \in Y .
$$

Let $\lambda_{n}(K)$ denote the $n$th largest eigenvalue of the nonnegative selfadjoint, compact linear operator $K$ on a Hilbert space. In this section, we will prove

THEOREM 3.1. Either

(i) $A$ is of finite rank, in which case there is an integer $n_{0} \geqslant 1$ for which

$$
\lambda_{n}\left(E^{*} A E\right)=\lambda_{n}(A)=0 \text { for } n \geqslant n_{0},
$$

or

(ii) $A$ is not of finite rank, in which case

$$
\lim _{n \rightarrow \infty} \lambda_{n}\left(E^{*} A E\right)=\lim _{n \rightarrow \infty} \lambda_{n}(A)=0
$$

and

$$
\lim _{n \rightarrow \infty} \frac{\lambda_{n}\left(E^{*} A E\right)}{\lambda_{n}(A)}=0 .
$$

Proof of (i): Let $\operatorname{rank}(A)=n_{0}-1$. Then $A$ has at most $n_{0}-1$ nonzero eigenvalues, so that $\lambda_{n}(A)=0$ for $n \geqslant n_{0}$. But

$$
\operatorname{rank}\left(E^{*} A E\right) \leqslant \operatorname{rank}(A E) \leqslant \operatorname{rank}(A)=n_{0}-1,
$$

so that $E^{*} A E$ has at most $n_{0}-1$ nonzero eigenvalues, i.e., $\lambda_{n}\left(E^{*} A E\right)=0$ for $n \geqslant n_{0}$.

Before proceeding to prove (ii) of Theorem 3.1, we must set up some machinery and prove two lemmas. Let $y_{1}, y_{2}, \ldots$ be an orthonormal family of eigenvectors for $A$, i.e., $A y_{j}=\lambda_{j}(A) y_{j}$, where $\lambda_{1}(A) \geqslant \lambda_{2}(A) \geqslant \cdots>0$ because $A$ is not of finite rank. Let

$$
M_{n}=s p\left\{y_{1}, \ldots, y_{n}\right\}^{\perp}={\overline{s p\left\{y_{j}\right\}_{j}}}_{j n+1} \oplus \operatorname{ker} A
$$

LEMMA 3.1. $\operatorname{codim} E^{-1} M_{n} \leqslant n$.

Proof. Let $L_{n}=X / s p\left\{E^{*} y_{j}\right\}_{j=1}^{n}=s p\left\{E^{*} y_{1}, \ldots, E^{*} y_{n}\right\}^{\perp}$ in $X$. Then $L_{n}=E^{-1} M_{n}$, since

$$
\begin{aligned}
x \in L_{n} & \Leftrightarrow x \perp\left\{E^{*} y_{1}, \ldots, E^{*} y_{n}\right\} \Leftrightarrow E x \perp\left\{y_{1}, \ldots, y_{n}\right\} \\
& \Leftrightarrow E x \in M_{n} \Leftrightarrow x \in E^{-1} M_{n} .
\end{aligned}
$$

So $\operatorname{codim} E^{-1} M_{n}=\operatorname{codim} L_{n} \leqslant n$. 
LEMMA 3.2. Let

$$
\rho_{n}=\sup _{\substack{i x \in M_{n} \\ 1 \pm E \\\|x\| \leqslant 1}}\|E x\|
$$

Then

$$
\frac{\lambda_{n+1}\left(E^{*} A E\right)}{\lambda_{n+1}(A)} \leqslant \rho_{n}^{2}
$$

Proof. Since $\operatorname{ker} A$ is a closed subspace of $M_{n}, E^{-1} \operatorname{ker} A$ is a closed subspace of $E^{-1} M_{n}$. Hence we have a direct sum decomposition

$$
E^{-1} M_{n}=E^{-1} \operatorname{ker} A \oplus\left[E^{1} M_{n} / E^{-1} \operatorname{ker} A\right] .
$$

Given $x \in E^{-1} M_{n},\|x\| \leqslant 1$, write

$$
x=x_{1}+x_{2} \quad\left(x_{1} \in E^{-1} \operatorname{ker} A, x_{2} \in E^{-1} M_{n} / E^{-1} \operatorname{ker} A\right),
$$

and note that $\left\|x_{2}\right\| \leqslant\|x\| \leqslant 1$. Then $A E x_{1}=0$ implies

$$
\begin{aligned}
\left(E^{*} A E x, x\right) & =\left(E^{*} A E x_{1}, x_{1}\right)+2\left(E^{*} A E x_{1}, x_{2}\right)+\left(E^{*} A E x_{2}, x_{2}\right) \\
& =\left(E^{*} A E x_{2}, x_{2}\right)=\left(A E x_{2}, E x_{2}\right) .
\end{aligned}
$$

If $E x_{2}=0$, then

$$
\left(A E x_{2}, E x_{2}\right)=0 \leqslant \lambda_{n+1}(A) \cdot 0=\lambda_{n+1}(A)\left\|E x_{2}\right\|^{2},
$$

while if $E x_{2} \neq 0$, set $y=E x_{2} /\left\|E x_{2}\right\|$ to find

$$
\left(A E x_{2}, E x_{2}\right)=(A y, y)\left\|E x_{2}\right\|^{2} \leqslant \lambda_{n+1}(A)\left\|E x_{2}\right\|^{2},
$$

since $y \in M_{n}$ and $\|y\| \leqslant 1$ implies $(A y, y) \leqslant \lambda_{n+1}(A)$. In either case. (3.6) or (3.7) yields

$$
\left(A E x_{2}, E x_{2}\right) \leqslant \lambda_{n+1}(A)\left\|E x_{2}\right\|^{2} .
$$

Now $E x_{2} \in M_{n}, x_{2} \perp E^{-1} \operatorname{ker} A$, and $\left\|x_{2}\right\| \leqslant 1$ yield

$$
\left\|E x_{2}\right\| \leqslant \rho_{n} \text {. }
$$

So (3.5), (3.8) and (3.9) yield

$$
\left(E^{*} A E x, x\right) \leqslant \lambda_{n+1}(A) \rho_{n}^{2} .
$$

Since the choice of $x \in E^{-1} M_{n} \cap B X$ is arbitrary, we have

$$
\sup _{\substack{x \in E ' M_{n} \\\|x\| \leqslant 1}}\left(E^{*} A E x, x\right) \leqslant \lambda_{n+1}(A) \rho_{n}^{2} .
$$

Since $\operatorname{codim} E^{-1} M_{n} \leqslant n$, we use the Courant minimax theorem to find

$$
\sup _{\substack{x \in E^{-1} M_{n} \\\|x\| \leqslant 1}}\left(E^{*} A E x, x\right) \geqslant \inf _{\substack{l, \subseteq K \\ \operatorname{codim} L \leqslant n}} \sup _{\substack{x \in L \\\|x\| \leqslant 1}}\left(E^{*} A E x, x\right)=\lambda_{n+1}\left(E^{*} A E\right) .
$$

The lemma follows from (3.11) and (3.12).

We are now ready to complete the

Proof of Theorem 3.1(ii): Since $A$ and $E^{*} A E$ are compact, the first statement is immediate. Now $M_{1} \supset M_{2} \supset M_{3} \supset \cdots$ and the definition of $\rho_{n}$ imply that

$$
\rho_{1} \geqslant \rho_{2} \geqslant \rho_{3} \geqslant \cdots \geqslant 0 \text {. }
$$


Hence there is a $\rho \geqslant 0$ such that

$$
\rho=\lim _{n \rightarrow \infty} \rho_{n}
$$

So Lemma 3.2 yields

$$
0 \leqslant \varlimsup_{n \rightarrow \infty} \frac{\lambda_{n}\left(E^{*} A E\right)}{\lambda_{n}(A)} \leqslant \rho^{2} .
$$

We need only show that $\rho=0$.

To this end, we choose a sequence $\left\{\varepsilon_{n} \geqslant 0\right\}_{n \geqslant 1}$ such that $\varepsilon_{n} \rightarrow 0$ as $n \rightarrow \infty$, and a sequence $\left\{x_{n}^{*} \in\left[E^{-1} M_{n} / E^{-1} \operatorname{ker} A\right] \cap B X\right\}_{n \geqslant 1}$ such that

$$
\left\|E x_{n}^{*}\right\| \leqslant \rho_{n} \leqslant\left\|E x_{n}^{*}\right\|+\varepsilon_{n} \text { for all } n \geqslant 1 \text {. }
$$

Then $\left\|x_{n}^{*}\right\| \leqslant 1$ implies that $x_{n}^{*}$ is weakly convergent (through some subsequence, say $\left\{n_{k}\right\}_{k \geqslant 1}$, of indices) to some $x^{*} \in B X$ (Theorem VIII.4.2 of Schechter [5]):

$$
x_{n_{k}}^{*} \rightarrow x^{*} \text { as } k \rightarrow \infty \text {. }
$$

Moreover, the compactness of $E$ implies that $E x_{n_{k}}^{*}$ converges strongly to $E x^{*}$ as $k \rightarrow \infty$ (see Theorem 5.1.1 of Friedman [3]). This implies that

$$
\lim _{k \rightarrow \infty}\left\|E x_{n_{k}}^{*}\right\|=\left\|E x^{*}\right\|
$$

and so (3.16) and (3.18) yield

$$
\rho=\left\|E x^{*}\right\| \text {. }
$$

We first claim that $x^{*} \perp E^{-1} \operatorname{ker} A$. Indeed, let $x \in E^{-1} \operatorname{ker} A$. Then $x_{n_{k}}^{*}-x^{*}$ as $k \rightarrow \infty$ and $x_{n_{k}}^{*} \perp E^{-1} \operatorname{ker} A$ imply

$$
\left(x^{*}, x\right)=\lim _{k}\left(x_{n_{k}}^{*}, x\right)=0 .
$$

Since $x \in E^{-1} \operatorname{ker} A$ is arbitrary, $x^{*} \perp E^{-1} \operatorname{ker} A$, as claimed.

We next claim that $x^{*} \in E^{-1} \operatorname{ker} A$, i.e., $E x^{*} \in \operatorname{ker} A$. Indeed, since $(\operatorname{ker} A)^{\perp}$ has the orthonormal basis $\left\{y_{j}\right\}_{j \geqslant 1}$, it suffices to show that

$$
\left(E x^{*}, y_{j}\right)=0 \text { for all } j \geqslant 1 \text {. }
$$

Given such an index $j$, choose $k_{0}$ such that $n_{k_{0}} \geqslant j$. Then for any $k \geqslant k_{0}$, we have $n_{h} \geqslant j$, so that $x_{n_{h}}^{*} \in E^{-1} M_{n_{h}}$ implies

$$
\left(x_{n_{k}}^{*}, E^{*} y_{j}\right)=\left(E x_{n_{k}}^{*}, y_{j}\right)=0 \text {. }
$$

By (3.17), we thus find

$$
\left(E x^{*}, y_{j}\right)=\left(x^{*}, E^{*} y_{j}\right)=\lim _{n \rightarrow \infty}\left(x_{n_{k}}^{*}, E^{*} y_{j}\right)=0 .
$$

Since the index $j \geqslant 1$ was arbitrary, it follows that $x^{*} \in E^{-1} \operatorname{ker} A$, as claimed.

So $x^{*} \in E^{-1} \operatorname{ker} A \cap\left(E^{-1} \operatorname{ker} A\right)^{\perp}=0$, implying

$$
\rho=\left\|E x^{*}\right\|=0 \text {, }
$$

completing the proof of the theorem.

In order to consider the seminormed case, we will need to know whether it is true that

$$
\lim _{n \rightarrow \infty} \frac{\lambda_{n}\left(E^{*} A E\right)}{\lambda_{n+m}(A)}=0
$$


for some positive integer $m$ ? In general, the answer is in the negative whenever $E$ is not of finite rank.

Indeed, let $x_{1}, x_{2}, \ldots \in X$ denote orthonormal eigenvectors of $E^{*} E$ corresponding to the eigenvalues $\varepsilon_{1}^{2} \geqslant \varepsilon_{2}^{2} \geqslant \cdots>0$, so that

$$
E^{*} E x_{n}=\varepsilon_{n}^{2} x_{n} \quad(n \geqslant 1) \text { and }\left(x_{m}, x_{n}\right)=\delta_{m n} \quad(m, n \geqslant 1) .
$$

Let

$$
y_{n}:=E x_{n} / \varepsilon_{n} \quad(n \geqslant 1),
$$

so that

$$
\left(y_{m}, y_{n}\right)=\frac{1}{\varepsilon_{m} \varepsilon_{n}}\left(E^{*} E x_{m}, x_{n}\right)=\frac{\varepsilon_{m}}{\varepsilon_{n}} \delta_{m n}=\delta_{m n} \quad(m, n \geqslant 1) \text {, }
$$

i.e., $y_{1}, y_{2}, \ldots$ form an orthonormal basis for

$$
M:={\overline{\operatorname{sp}\left\{y_{n}\right\}}}_{n \geqslant 1} \text {. }
$$

Now define $A: Y \rightarrow Y$ as follows. Let $\alpha_{1} \geqslant \alpha_{2} \geqslant \cdots>0$, with $\lim _{n \rightarrow \infty} \alpha_{n}=0$ be given. For any $y \in Y$, there exists a unique choice of scalars $\eta_{1}, \eta_{2}, \ldots$ and $y_{0} \in M^{\perp}$ such that

$$
y=\sum_{n=1}^{\infty} \eta_{n} y_{n}+y_{0} .
$$

Then let

$$
A y:=\sum_{n=1}^{\infty} \eta_{n} \alpha_{n} y_{n} .
$$

We then see that $y_{1}, y_{2}, \ldots$ are orthonormal eigenvectors of $A$ corresponding to the eigenvalues $\alpha_{1}, \alpha_{2}, \ldots$, so that

$$
\lambda_{n}(A)=\alpha_{n} .
$$

Moreover, let $x \in X$, so that there exists a unique choice of scalars $\xi_{1}, \xi_{2}, \ldots$ and $x_{0} \in \operatorname{ker} E^{*} E$ such that

$$
x=\sum_{n=1}^{\infty} \xi_{n} x_{n}+x_{0}
$$

we claim that

$$
E^{*} A E x=\sum_{n=1}^{\infty} \xi_{n} \alpha_{n} \varepsilon_{n}^{2} x_{n}
$$

which implies that $x_{1}, x_{2}, \ldots$ are orthonormal eigenvectors of $E^{*} A E$ corresponding to the eigenvalues $\alpha_{1} \varepsilon_{1}^{2} \geqslant \alpha_{2} \varepsilon_{2}^{2} \geqslant \cdots>0$, so that

$$
\lambda_{n}\left(E^{*} A E\right)=\alpha_{n} \varepsilon_{n}^{2} .
$$

Indeed, let $x \in X$ have the representation (3.33). Then $E x_{0} \in M^{\perp}$, since for any index $n \geqslant 1$,

$$
\left(E x_{0}, y_{n}\right)=\frac{1}{\varepsilon_{n}}\left(E x_{0}, E x_{n}\right)=\frac{1}{\varepsilon_{n}}\left(E^{*} E x_{0}, x_{n}\right)=0
$$


Thus (3.33) implies that $E x_{0} \in M^{\perp}$ in the representation

$$
E x=\sum_{n=1}^{\infty} \xi_{n} E x_{n}+E x_{0}=\sum_{n=1}^{\infty} \xi_{n} \varepsilon_{n} y_{n}+E x_{0},
$$

and thus (3.30) and (3.31) imply

$$
A E x=\sum_{n=1}^{\infty} \xi_{n} \varepsilon_{n} \alpha_{n} y_{n}=\sum_{n=1}^{\infty} \xi_{n} \alpha_{n} E x_{n} .
$$

So

$$
E^{*} A E x=\sum_{n=1}^{\infty} \xi_{n} \alpha_{n} E^{*} E x_{n}=\sum_{n=1}^{\infty} \xi_{n} \alpha_{n} \varepsilon_{n}^{2} x_{n}
$$

as claimed.

We now show that not only is (3.25) false in general, but the limit can be any positive number, or can go to infinity arbitrarily fast.

THEOREM 3.2. Given a compact linear $E: X \rightarrow Y$, a positive integer $m$, and $a$ sequence $\mu_{1} \leqslant \mu_{2} \leqslant \cdots$ of positive real numbers, there exists a compact, linear, nonnegative, selfadjoint $A: Y \rightarrow Y$ such that

$$
\frac{\lambda_{n}\left(E^{*} A E\right)}{\lambda_{n+m}(A)}=\mu_{n}
$$

for all sufficiently large $n$. Hence, for any $\mu \in[0, \infty]$, there exists an $A$ for which

$$
\lim _{n \rightarrow \infty} \frac{\lambda_{n}\left(E^{*} A E\right)}{\lambda_{n+m}(A)}=\mu,
$$

and if $\mu=\infty$, the limit can go to infinity arbitrarily fast.

Proof. Let $E,\left\{\mu_{n}\right\}_{n \geqslant 1}$, and $m$ be as in the statement of the theorem. Let $E^{*} E$ be as in (3.26), and let $A$ be defined by (3.30), (3.26), where now

$$
\alpha_{n}:=\prod_{i=0}^{k-1} \frac{\varepsilon_{i m+j}^{2}}{\mu_{i m+j}} \quad\left(k:=\left\lfloor\frac{n-1}{m}\right\rfloor, j=n-k m\right) .
$$

Then there is a positive integer $n_{0}$ such that (3.32) and (3.35) hold for all $n \geqslant n_{0}$. Hence for $n \geqslant n_{0}$, let $k=\lfloor(n-1) / m\rfloor$ and $j=n-k m$ to find

$$
\frac{\lambda_{n}\left(E^{*} A E\right)}{\lambda_{n+m}(A)}=\frac{\alpha_{n} \varepsilon_{n}^{2}}{\alpha_{n+m}}=\frac{\alpha_{k m+j} \varepsilon_{k m+j}^{2}}{\alpha_{(k+1) m+j}}=\mu_{k m+j}=\mu_{n},
$$

proving the first statement. The second statement follows by taking $\mu \in(0, \infty)$ to be the limit of the $\mu_{n}$ as $n \rightarrow \infty$. To make the second statement hold for $\mu=0$, take $\gamma \in(0,1)$ and let

$$
\alpha_{n}:=\gamma^{n}
$$

Then (3.32) and (3.35) hold for all $n \geqslant 1$. So

$$
\frac{\lambda_{n}\left(E^{*} A E\right)}{\lambda_{n+m}(A)}=\frac{\alpha_{n} \varepsilon_{n}^{2}}{\alpha_{n+m}}=\gamma^{-m} \varepsilon_{n}^{2} \rightarrow 0 \quad \text { as } n \rightarrow \infty .
$$


4. Regularity and Optimal Error in the Normed Case. We now consider the case where we have Hilbert spaces $\bar{v}_{1}, \bar{T}_{1}$, and $\bar{v}_{2}$ where there is a compact transformation $E: \bar{w}_{1} \rightarrow \bar{T}_{1}$, so that when $E$ is injective, $E\left(\bar{\sigma}_{1}\right)$ may be identified as a subspace of $\bar{T}_{1}$ which has more "regularity" than the space $" \bar{T}_{1}$. Let $\bar{T}_{0}$ and $\bar{T}_{0}$ denote the unit balls of $\bar{r}_{1}$ and $\bar{F}_{1}$, respectively. Suppose that $S: \bar{r}_{1} \rightarrow \bar{r}_{2}$ is a bounded linear solution operator; then we define the solution operator $\tilde{S}: \tilde{r}_{1} \rightarrow \bar{r}_{2}$ by $\tilde{S}=S E$. We now consider the problems given by $S$ with $\bar{T}_{0}$ and $\tilde{S}$ with $\bar{x}_{0}$.

Our first result shows that for the normed case, replacing the problem $\left(S,{ }^{\circ} \bar{F}_{0}\right)$ by the problem $\left(\tilde{S}, \tilde{F}_{0}\right)$ does not increase the $n$th minimal radius beyond a factor of $\|E\|$.

THEOREM 4.1. For all $n \geqslant 0$,

$$
r\left(n, \dot{S}, \tilde{T}_{0}\right) \leqslant\|E\| r\left(n, S, \widetilde{T}_{0}\right) \text {. }
$$

Proof. If $E=0$, then $\tilde{S}=S E=0$ and $\|E\|=0$, so that the inequality reduces to $0 \leqslant 0$. We now suppose that $E \neq 0$. Let $) r$ be an information operator on $\bar{T}_{1}$ of cardinality at most $n$. Define an information operator $\bar{r}$ on $\tilde{\bar{r}}$ by $: \tilde{r}:=$ Then $\# \tilde{r} \leqslant \# \leqslant n$, so that

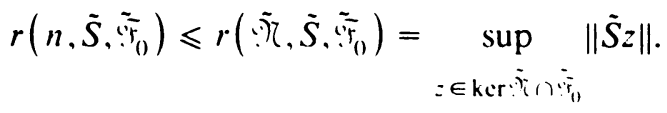

Let $z \in \operatorname{ker} \bar{\tau} \cap \bar{r}_{0}$. Set $y=E z /\|E\|$. Then

$$
\because y=\frac{1}{\|E\|} \cdot r E z=\frac{1}{\|E\|} \cdot \tilde{r}_{z}=0 \text {. }
$$

so that $y \in \operatorname{ker}: x \cap \bar{T}_{0}$. So

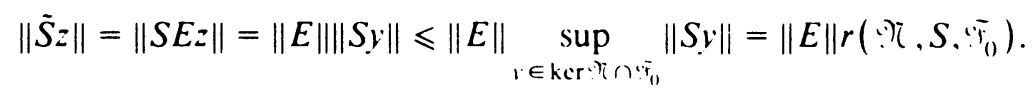

Taking the sup over all such $z$ and using (4.1), we have

$$
r\left(n, \tilde{S}, \tilde{\bar{v}}_{0}\right) \leqslant\|E\| r\left(\because, S, \bar{r}_{0}\right) \text {. }
$$

Since $: \pi$ is an arbitrary information operator on ${ }^{2} \bar{r}$, of cardinality at most $\because \pi$, we may take the inf over all such $\mathrm{r}$ to complete the proof of the theorem.

Note that this result is nonasymptotic, holding for all $n \geqslant 0$. We now give an asymptotic result which says that in the limit, replacing $\left(S,\left(\bar{T}_{0}\right)\right.$ with $\left(\tilde{S}, \tilde{T}_{0}\right)$ helps beyond any positive factor, no matter how small.

\section{THEOREM 4.2. Either}

(i) $S$ is bounded but not compact, in which case there exists $\rho>0$ such that

$$
\lim _{n \rightarrow \infty} r\left(n, S, \bar{y}_{0}\right)=\rho \text { and } \lim _{n \rightarrow \infty} r\left(n, \dot{S}, \tilde{\pi}_{0}\right)=0 \text {. }
$$

or

(ii) $S$ is of finite rank, in which case there is an integer $n_{0}$ such that

$$
r\left(n, S, \bar{v}_{0}\right)=r\left(n, \tilde{S}, \tilde{\bar{v}}_{0}\right)=0 \text { for } n \geqslant n_{0},
$$

or

(iii) $S$ is compact, but not of finite rank, in which case

$$
\lim _{n \rightarrow \infty} r\left(n, S, \widetilde{\mathscr{F}}_{0}\right)=\lim _{n \rightarrow \infty} r\left(n, \tilde{S}, \tilde{\mathscr{F}}_{0}\right)=0 \quad \text { and } \lim _{n \rightarrow \infty} \frac{r\left(n, \tilde{S}, \tilde{\mathscr{F}}_{0}\right)}{r\left(n, S, \widetilde{\mathscr{F}}_{0}\right)}=0 .
$$


Proof. For part (i), let $S$ be bounded and noncompact. Then Corollary 2.5.1 of [6] implies that there is a $\rho_{0}>0$ such that $r\left(n, S, \mathscr{F}_{0}\right) \geqslant \rho_{0}$. Since $r\left(n, S, \mathscr{F}_{0}\right)$ is monotonically nonincreasing, the first statement in (i) follows. On the other hand, $S$ bounded and $E$ compact imply $\tilde{S}=S E$ is compact. Thus Corollary 2.5.1 of [6] yields that $r\left(n, \tilde{S}, \widetilde{F}_{0}\right)$ converges to zero.

For parts (ii) and (iii), let $X=\mathscr{F}_{1}, Y=\mathscr{F}_{2}, A=S^{*} S$. Then $A=A^{*} \geqslant 0$ and $E$ are compact. Since the restriction map is the identity, it has trivial kernel, and so the indices of the problems $\left(S, \mathscr{F}_{0}\right)$ and $\left(\tilde{S}, \tilde{F}_{0}\right)$ are zero. Thus $(2.18)$ yields

$$
r\left(n, S, \mathscr{F}_{0}\right)=\lambda_{n+1}^{1 / 2}(A) \text { and } r\left(n, \tilde{S}, \tilde{\mathscr{F}}_{0}\right)=\lambda_{n+1}^{1 / 2}\left(E^{*} A E\right)
$$

The result now follows immediately from Theorem 3.1.

We now show how increased regularity improves optimal error behavior in the normed case. Consider a well-posed (i.e. bounded) linear problem $S$ defined on $H^{r}(\Omega)$, where $\Omega$ is a smooth bounded region in $\mathbf{R}^{p}$. Thus there is a Hilbert space $Z$ such that $S: H^{r}(\Omega) \rightarrow Z$ is a bounded linear operator. We pick $s>r$ and let $E$ : $H^{s}(\Omega) \rightarrow H^{r}(\Omega)$ denote the inclusion injection, i.e., $E f:=f$ for $f \in H^{s}(\Omega)$. Setting $\tilde{S}:=S E$ (i.e., $\tilde{S}$ is $S$ restricted to $H^{s}(\Omega)$ ), we let

$$
e(n, r):=r\left(n, S, B H^{r}(\Omega)\right)
$$

and

$$
e(n, s):=r\left(n, \tilde{S}, B H^{s}(\Omega)\right)
$$

denote the minimal errors of algorithms using information of cardinality at most $n$ when the admissible inputs are the unit balls of $H^{r}(\Omega)$ and $H^{s}(\Omega)$, respectively.

THEOREM 4.3. For all $n \geqslant 0$,

$$
e(n, s) \leqslant e(n, r) \text {. }
$$

Moreover, precisely one of the following statements holds:

(i) $S$ is bounded, but not compact, in which case, there exists $\varepsilon>0$ such that

$$
\lim _{n \rightarrow \infty} e(n, r)=\varepsilon
$$

while

$$
\lim _{n \rightarrow \infty} e(n, s)=0
$$

or

(ii) $S$ is of finite rank, in which case there is an integer $n_{0}$ such that

$$
e(n, r)=e(n, s)=0 \text { for } n \geqslant n_{0},
$$

or

(iii) $S$ is compact, but not of finite rank, in which case

$$
\lim _{n \rightarrow \infty} e(n, r)=\lim _{n \rightarrow \infty} e(n, s)=0 \text { and } \lim _{n \rightarrow \infty} \frac{e(n, s)}{e(n, r)}=0 \text {. }
$$

Proof. Let $\tilde{F}_{1}=H^{r}(\Omega)$ and $\tilde{\mathscr{F}}_{1}=H^{s}(\Omega)$, so that $\mathscr{F}_{0}=B H^{r}(\Omega)$ and $\tilde{\mathscr{F}}_{0}=B H^{s}(\Omega)$. Since $\|\cdot\|_{r} \leqslant\|\cdot\|_{s}$, we have $\|E\| \leqslant 1$. Hence the first statement follows from Theorem 4.1. By the Kondrasov lemma (see, e.g., p. 114 of [2]), $E$ is compact. Thus the second statement follows from Theorem 4.2 . 
Thus either the problem can be solved exactly using a finite amount of information (case (ii)) or increasing the smoothness of the problem by assuming the existence of additional derivatives improves the optimal error by more than any fixed constant factor as $n \rightarrow \infty$ (cases (i) and (iii)).

Remark 4.1. If we replace $H^{r}(\Omega)$ and $H^{s}(\Omega)$ in (4.1) and (4.2) by $H_{0}^{r}(\Omega)$ and $H_{0}^{s}(\Omega)$, Theorem 4.2 still holds, since the inclusion injection $E_{0}: H_{0}^{s}(\Omega) \rightarrow H_{0}^{r}(\Omega)$ is compact for $r<s$.

5. Regularity and Optimal Error in the Seminormed Case. We now consider the case when regularity is measured by a Sobolev seminorm (as was the situation in the examples studied in [6]). In this section, we show that Theorem 4.3 does not hold when the unit balls $B H^{r}(\Omega)$ and $B H^{s}(\Omega)$ are replaced by the unit semiballs $4 B H^{r}(\Omega)$ and $(1) H^{s}(\Omega)$ (see $(2.3)$ ). In fact, we show that there is a penalty associated with increasing the regularity in this manner. and that this penalty can be arbitrarily big. On the other hand, we are able to show that a slight modification of Theorem 4.3 does hold in the seminormed case, and we give sufficient conditions for the original version of this theorem to hold in this case.

We now let $Z$ be a Hilbert space and let $S: H^{r}(\Omega) \rightarrow Z$ be a bounded linear solution operator, where $\Omega \subset \mathbf{R}^{p}$ is a smooth, bounded, simply connected region. We consider the problem defined by $S$ and ${ }^{\circ} \bar{F}_{0}:=S_{1} H^{r}(\Omega)$.

It will be useful to write $\bar{r}_{0}$ in terms of a restriction operator $T$. Let $\mu_{1}, \ldots, \mu_{m}$ denote the multi-indices $\mu$ in $p$ variables such that $|\mu|=r$, so that

$$
m=\left(\begin{array}{c}
p+r-1 \\
r
\end{array}\right) \text {. }
$$

Consider the Hilbert space $L_{2}(\Omega)^{m}$. and define a subspace $V$ of $L_{2}(\Omega)^{m}$ by

$$
\left[g_{1} \cdots g_{m}\right]^{T} \in V \quad \text { iff } \exists f \in H^{r}(\Omega): D^{\mu} \cdot f=g_{1}(1 \leqslant i \leqslant m) .
$$

LEMMA 5.1. $V$ is closed in $L_{2}(\Omega)^{m}$.

Proof. Since $\Omega$ is simply connected, $g \in V$ if and only if $g$ satisfies a set of $q$ equations in $H^{-1}(\Omega)$ of the form

$$
\partial_{1} g_{1}-\partial_{k} g_{l}=0
$$

where $i, j, k, l$ are related by the relation

$$
\partial_{i} D^{\mu_{l}}=\partial_{k} D^{\mu_{\prime}},
$$

which expresses the equality of the mixed partial derivatives of the function $f$ for which (5.2) holds. Hence there is a bounded linear operator $L: L_{2}(\Omega)^{m} \rightarrow H^{-1}(\Omega)^{q}$ such that $V=\operatorname{ker} L$, and so $V$ is closed.

Hence $V$ is a Hilbert subspace of $L_{2}(\Omega)^{m}$. We now define $T: H^{r}(\Omega) \rightarrow V$ by

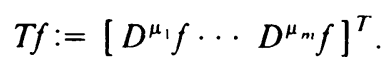

By (5.2), $T$ is a surjection.

In what follows, it will be useful to have another characterization of the index of the problem.

LemMa 5.2. The index $n^{*}(T, S)$ of the problem $\left(S, \bar{y}_{0}\right)$ is given by

$$
n^{*}=n^{*}(T, S)=\operatorname{dim} S(\operatorname{ker} T) \text {. }
$$


Proof. First note that

$$
\operatorname{ker}\left(\left.S\right|_{\text {ker } T}\right)=\operatorname{ker} T \cap \operatorname{ker} S .
$$

Since $\operatorname{dim} \operatorname{ker} T$ is finite, we let $M=\operatorname{ker} T$ in the equation

$$
\operatorname{dim} S(M)+\operatorname{dim}\left(\left.\operatorname{ker} S\right|_{M}\right)=\operatorname{dim} M .
$$

Using (5.7) and (2.17), we find

$$
\begin{aligned}
\operatorname{dim} S(\operatorname{ker} T) & =\operatorname{dim}(\operatorname{ker} T)-\operatorname{dim}\left(\left.\operatorname{ker} S\right|_{\operatorname{ker} T}\right) \\
& =\operatorname{dim}(\operatorname{ker} T)-\operatorname{dim}(\operatorname{ker} T \cap \operatorname{ker} S)=n^{*}(T, S) .
\end{aligned}
$$

In order to proceed, we let $P_{t}(\Omega)$ denote the space of polynomials of degree $t$ on $\Omega$. (Note that $P_{r-1}(\Omega)$ is the kernel of $T$ given by (5.5).) Let

$$
\hat{H}^{r}(\Omega):=H^{r}(\Omega) / P_{r-1}(\Omega)
$$

denote the orthogonal complement of $P_{r-1}(\Omega)$ in $H^{r}(\Omega)$, so that

$$
f \in \hat{H}^{r}(\Omega) \text { iff } f \in H^{r}(\Omega) \text { and }(f, p)_{r}=0 \forall p \in P_{r-1}(\Omega),
$$

where $(\cdot, \cdot)_{r}$ is the inner product which yields the $\|\cdot\|_{r}$-norm. By Theorem 3.1.1 of [2], the $H^{r}(\Omega)$-seminorm $|\cdot|_{r}$ is a norm on $\hat{H}^{r}(\Omega)$, equivalent to the usual quotient norm $\|\cdot\|_{r}$ on $\hat{H}^{r}(\Omega)$ given by

$$
\|f\|_{r}:=\inf _{p \in P_{r-1}(\Omega)}\|f+p\|_{r} .
$$

The problem $\left(S, \Re H^{r}(\Omega)\right)$ now induces a new problem $\left(\hat{S}, \hat{\mathscr{F}}_{0}\right)$ by letting $\hat{S}$ : $\hat{H}^{r}(\Omega) \rightarrow Z$ be defined by

$$
\hat{S} f:=S f \text { for } f \in \hat{H}^{r}(\Omega)
$$

and letting

$$
\hat{\mathscr{F}}_{0}:=B \hat{H}^{r}(\Omega)=\mathscr{F}_{0} \cap \hat{H}^{r}(\Omega)
$$

LEMMA 5.3. $r\left(n+n^{*}, S, \mathscr{F}_{0}\right)=r\left(n, \hat{S}, \hat{\mathscr{F}}_{0}\right)$.

Proof. We first let $\hat{\mathfrak{T}}: \hat{H}^{r}(\Omega) \rightarrow \mathbf{R}^{n}$ be a linear information operator of cardinality at most $n$. Define $\Re: H^{r}(\Omega) \rightarrow \mathbf{R}^{n}$ by

$$
\Re f:=\left[\begin{array}{l}
S \hat{p} \\
\hat{\vartheta} \hat{f}
\end{array}\right],
$$

where $\hat{f} \in \hat{H}^{r}(\Omega)$ and $\hat{p} \in P_{r-1}(\Omega)$ are uniquely chosen so that

$$
f=\hat{f}+\hat{p} \text {. }
$$

Then Lemma 5.2 yields

$$
\# \mathfrak{\Re} \leqslant \# \hat{\mathfrak{X}}+\operatorname{dim} S\left(P_{r-1}(\Omega)\right) \leqslant n+n^{*} .
$$

We claim that $f \in \operatorname{ker} \mathscr{U} \cap \mathscr{F}_{0}$ implies $\hat{f} \in \operatorname{ker} \hat{\mathfrak{R}} \cap \hat{\mathscr{F}}_{0}$ and $S f=\hat{S} \hat{f}$. Indeed, given such an $f$, write $f=\hat{f}+\hat{p}$ as in (5.16). Then $f \in \mathscr{F}_{0}$ implies

$$
\|\hat{f}\|_{r}^{2} \leqslant\|\hat{f}\|_{r}^{2}+\|\hat{p}\|_{r}^{2}=\|f\|_{r}^{2} \leqslant 1,
$$

so that $\hat{f} \in \hat{\mathscr{F}}_{0}$. Moreover $f \in \operatorname{ker} \mathfrak{N}$ implies

$$
\hat{\mathfrak{Y}} \hat{f}=0 \quad \text { and } \quad S \hat{p}=0
$$


i.e., $\hat{f} \in \operatorname{ker} \hat{\mathfrak{K}}$ and

$$
S f=S \hat{f}+S \hat{p}=S \hat{f}=\hat{S} \hat{f}
$$

proving the claim.

From (2.14) and (5.17), we find

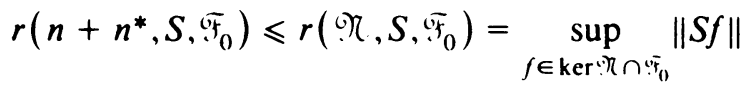

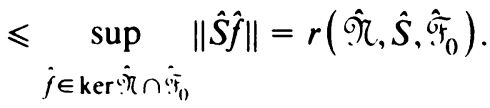

Taking the infimum over all $\hat{\mathfrak{Y}}$ of cardinality at most $n$, we find

$$
r\left(n+n^{*}, S,{\widetilde{F_{0}}}_{0}\right) \leqslant r\left(n, \hat{S}, \hat{\widetilde{F}}_{0}\right) \text {. }
$$

In order to prove that the inequality (5.22) is an equality, we consider an $\left(n+n^{*}\right)$ th optimal information for $\left(S, \widetilde{F}_{0}\right)$. By $(2.4 .12)$ of [6], this information has the form

$$
\Re_{n+n^{*}} f:=\left[\begin{array}{l}
\mathfrak{\varkappa}^{*} f \\
\hat{\mathfrak{X}}_{n} f
\end{array}\right]
$$

where

$$
\operatorname{ker} \Re^{*}=(\operatorname{ker} T \cap \operatorname{ker} S) \oplus(\operatorname{ker} T)^{\perp}
$$

and there is a linear transformation $L$ on $V$, of rank at most $n$, such that

$$
\hat{\vartheta}_{n}=L T \text {. }
$$

Let $\Pi: H^{r}(\Omega) \rightarrow P_{r-1}(\Omega)$ denote the orthogonal projector, so that $\hat{p}=\mathrm{II} f$ in (5.16). Claim there exists an invertible $n^{*} \times n^{*}$ matrix $M$ such that

$$
\Re *=M S \Pi \text {. }
$$

To do this, we first show that

$$
\operatorname{ker} \mathfrak{x}^{*} \subset \operatorname{ker} S \Pi \text {. }
$$

To see this, let $f \in \operatorname{ker} \Re^{*}$. By (5.24), we may write

$$
f=f_{1}+f_{2} \quad\left(f_{1} \in \operatorname{ker} T \cap \operatorname{ker} S, f_{2} \in(\operatorname{ker} T)^{\perp}\right) .
$$

Then $f_{1} \in \operatorname{ker} T$ implies $f_{1} \in P_{r-1}(\Omega)$. Since $\Pi$ is a projection onto $P_{r-1}(\Omega)$, we have $\Pi f_{1}=f_{1}$. Since $f_{1} \subset \operatorname{ker} S$, we have

$$
S \Pi f_{1}=S f_{1}=0 .
$$

On the other hand, $f_{2} \in(\operatorname{ker} T)^{\perp}=\hat{H}^{r}(\Omega)$ implies that $\Pi f_{2}=0$. So

$$
S \Pi f_{2}=0 \text {. }
$$

Hence $f \in \operatorname{ker} S \Pi$, proving (5.27). Since ker $\mathcal{X}^{*} \subset \operatorname{ker} S \Pi$ and

$$
\text { codimker } \varkappa^{*}=n^{*}=\operatorname{dim} \operatorname{range} S \Pi=\operatorname{codim} k e r S \Pi,
$$

Lemma 2.2.1 of [6] yields (5.26), as claimed.

So

$$
\Re_{n+n^{*}} f=\left[\begin{array}{c}
M S \Pi f \\
\hat{\Re}_{n} f
\end{array}\right]=\left[\begin{array}{c}
M S \hat{p} \\
\hat{\Re}_{n} \hat{f}
\end{array}\right],
$$


where $\hat{f}$ and $\hat{p}$ are given by (5.16) and $\hat{\mathfrak{T}}_{n} f=\hat{\mathfrak{T}}_{n} \hat{f}$ because $\hat{p} \in \operatorname{ker} T$ implies $L T f=L T \hat{f}$.

We now consider the information operator $\hat{\mathscr{X}}_{n}$ for the problem $\left(\hat{S}, \hat{\mathscr{F}}_{0}\right)$. Clearly $\# \hat{\mathfrak{X}}_{n} \leqslant n$. We claim that $\operatorname{ker} \hat{\mathscr{T}}_{n} \cap \hat{\mathscr{F}}_{0} \subset \operatorname{ker} \mathcal{X}_{n+n^{*}} \cap \mathscr{F}_{0}$. Indeed, let $f \in \operatorname{ker} \hat{\mathscr{T}}_{n} \cap$ $\hat{\mathscr{F}}_{0}$. Then $f \in \hat{\mathscr{F}}_{0}=\hat{H}^{r}(\Omega) \cap \mathscr{F}_{0}$ implies $f \in \mathscr{F}_{0}$. Moreover $f \in \hat{H}^{r}(\Omega)$ implies that $\hat{p}=0$ and $\hat{f}=f$ in (5.16), so that $f \in \operatorname{ker} \hat{\mathfrak{X}}_{n}$ implies

$$
\Re_{n+n^{*}} f=\left[\begin{array}{c}
M S 0 \\
\hat{\Re}_{n} f
\end{array}\right]=0 \text {, }
$$

establishing the claim.

Since \# $\hat{\Upsilon}_{n} \leqslant n$, we use the claim above and the fact that $\hat{S}=\left.S\right|_{\hat{H}^{r}(\Omega)}$ to find

$$
\begin{aligned}
& r\left(n, \hat{S}, \hat{\mathscr{F}}_{0}\right) \leqslant r\left(\hat{\mathscr{T}}_{n}, \hat{S}, \hat{\mathscr{F}}_{0}\right)=\sup _{f \in \hat{\mathscr{F}}_{0} \cap \operatorname{ker} \hat{\mathscr{\vartheta}}_{n}}\|\hat{S} f\| \\
& \leqslant \sup _{f \in \bar{F}_{0} \cap \operatorname{ker} \Re_{n+n^{*}}}\|S f\|=r\left(\Re_{n+n^{*}}, S, \mathscr{F}_{0}\right)=r\left(n+n^{*}, S, \mathscr{F}_{0}\right),
\end{aligned}
$$

the last by the optimality of $\Re_{n+n^{*}}$.

We wish to examine the effects of increasing regularity when $\mathscr{F}_{0}$ is the unit semiball of a Sobolev space. Recall that $Z$ is a Hilbert space, and that $S: H^{r}(\Omega) \rightarrow Z$ is a bounded linear transformation. Choose $s>r$, and let $E: H^{s}(\Omega) \rightarrow H^{r}(\Omega)$ denote the (compact) inclusion injection, as in Section 4.

LEMMA 5.4. $\hat{H}^{s}(\Omega)$ is a subspace of $\hat{H}^{r}(\Omega)$, and the inclusion injection $\hat{E}: \hat{H}^{s}(\Omega) \rightarrow$ $\hat{H}^{r}(\Omega)$ is compact.

Proof. To show that $\hat{H}^{s}(\Omega)$ is a subspace of $\hat{H}^{r}(\Omega)$, let $f \in \hat{H}^{s}(\Omega)$. Then $f \in H^{s}(\Omega)$ $\subset H^{r}(\Omega)$, and $(f, p)_{s}=0$ for $p \in P_{s-1}(\Omega)$. Now for any $p \in P_{r-1}(\Omega)$, we have $D^{\mu} p=0$ for $|\mu|>r$. So

$$
(f, p)_{r}=(f, p)_{s}-\sum_{r<|\mu| \leqslant s}\left(D^{\mu} f, D^{\mu} p\right)_{0}=(f, p)_{s}=0
$$

since $p \in P_{r-1}(\Omega) \subset P_{s-1}(\Omega)$ and $f \in \hat{H}^{s}(\Omega)=P_{s-1}(\Omega)^{\perp}$. Hence $f \in \hat{H}^{r}(\Omega)$.

We now show that $\hat{E}$ is compact. Let $\left\{f_{j}\right\}_{j=1}^{\infty} \subset \hat{H}^{s}(\Omega)$ be bounded, say

$$
\left|f_{j}\right|_{s} \leqslant M \quad(j \geqslant 1) \text {. }
$$

Since $f_{j} \in \hat{H}^{s}(\Omega)=P_{s-1}(\Omega)^{\perp}$, we find

$$
\left\|f_{j}\right\|_{s}=\inf _{p \in P_{s-1}(\Omega)}\left\|f_{j}+p\right\|_{s}=\left\|f_{j}\right\|_{s} \leqslant C\left|f_{j}\right|_{s} \leqslant C M,
$$

where the first inequality follows from the equivalence of $|\cdot|_{s}$ and $\|\cdot\|_{s}$ over $\hat{H}^{s}(\Omega)$. Since $r<s$, the Kondrasov lemma yields $g \in H^{r}(\Omega)$ and a subsequence $\left\{j_{k}\right\}$ such that $f_{j_{h}} \rightarrow g$ in $H^{r}(\Omega)$. Since $H^{r}(\Omega)=\hat{H}^{r}(\Omega) \oplus P_{r-1}(\Omega)$, there exists $f \in \hat{H}^{r}(\Omega)$ and $p \in P_{r-1}(\Omega)$ such that

$$
g=f+p
$$

We claim that $f_{j_{k}} \rightarrow f$ in $\hat{H}^{r}(\Omega)$. Indeed, $p \in P_{r-1}(\Omega)$ implies

$$
\begin{aligned}
\left|f_{j_{k}}-f\right|_{r} & =\left|f_{j_{k}}-g-p\right|_{r} \leqslant\left|f_{j_{k}}-g\right|_{r}+|p|_{r} \\
& =\left|f_{j_{k}}-g\right|_{r} \leqslant\left\|f_{j_{k}}-g\right\|_{r},
\end{aligned}
$$

so that $f_{j_{k}} \rightarrow g$ in $H^{r}(\Omega)$ establishes the claim and the lemma. 
Let $\tilde{S}=S E$ as in Section 4. Then the commutative diagram

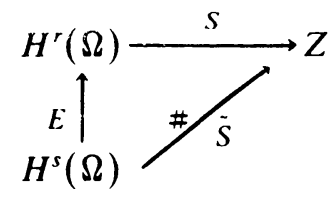

induces a diagram

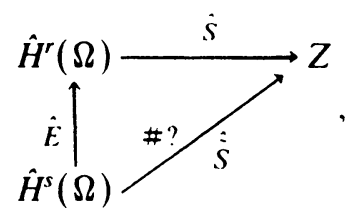

where $\hat{S}$ and $\hat{\tilde{S}}$ are the restrictions of $S$ and $\tilde{S}$ to $\hat{H}^{r}(\Omega)$ and $\hat{H}^{s}(\Omega)$, respectively (see (5.13)). We claim that the diagram (5.41) commutes. Indeed, let $f \in \hat{H}^{s}(\Omega)$. Then $\tilde{S}=S E$ yields

$$
\hat{\tilde{S}} f=\tilde{S} f=S E f=S f .
$$

On the other hand $f \in \hat{H}^{s}(\Omega) \subset \hat{H}^{r}(\Omega)$, so that (5.42) implies

$$
\hat{S} \hat{E} f=\hat{S} f=S f=\hat{\tilde{S}} f .
$$

Thus $\hat{S} \hat{E}=\hat{\tilde{S}}$, i.e., the diagram (5.41) commutes, as claimed.

We now are ready to discuss how the behavior of the optimal error changes when regularity is increased. Let $s>r$, let $n_{r}^{*}$ and $n_{s}^{*}$, respectively, denote the indices for the problems $\left(S, \mathscr{B} H^{r}(\Omega)\right)$ and $\left(\tilde{S}, \mathscr{Q} B H^{s}(\Omega)\right)$, let

$$
e(n, r):=r\left(n, S, \cup B H^{r}(\Omega)\right),
$$

and let

$$
e(n, s):=r\left(n, \tilde{S}, \mathscr{B} H^{s}(\Omega)\right)
$$

We then have the following modification of Theorem 4.3:

THEOREM 5.1. Precisely one of the following statements holds:

(i) $S$ is bounded, but not compact, in which case, there exists $\varepsilon>0$ such that

$$
\lim _{n \rightarrow \infty} e(n, r)=\varepsilon
$$

while

$$
\lim _{n \rightarrow \infty} e(n, s)=0
$$

or

(ii) $S$ is of finite rank, in which case, there is an integer $n_{0}$ such that

$$
e\left(n+n_{s}^{*}, s\right)=e\left(n+n_{r}^{*}, r\right)=0 \text { for } n \geqslant n_{0},
$$

or

(iii) $S$ is compact, but not of finite rank, in which case,

$$
\lim _{n \rightarrow \infty} e(n, r)=\lim _{n \rightarrow \infty} e(n, s)=0 \text { and } \lim _{n \rightarrow \infty} \frac{e\left(n+n_{s}^{*}, s\right)}{e\left(n+n_{r}^{*}, r\right)}=0 .
$$


Proof. Using (5.44) and Lemma 5.3, we have

$$
e\left(n+n_{r}^{*}, r\right)=r\left(n+n_{r}^{*}, S, \mathscr{B} H^{r}(\Omega)\right)=r\left(n, \hat{S}, B \hat{H}^{r}(\Omega)\right),
$$

while (5.45), Lemma 5.3, and (5.41) yield

$$
\begin{aligned}
e\left(n+n_{s}^{*}, s\right) & =r\left(n+n_{s}^{*}, \tilde{S}, \Re H^{s}(\Omega)\right)=r\left(n, \hat{\tilde{S}}, B \hat{H}^{s}(\Omega)\right) \\
& =r\left(n, \hat{S} \hat{E}, B \hat{H}^{s}(\Omega)\right) .
\end{aligned}
$$

The result now follows from (5.46), (5.47), Lemma 5.4, and Theorem 4.2.

As a corollary, we can give sufficient conditions for Theorem 4.3 to hold:

COROLlaRY 5.1. Suppose that either

$$
n_{s}^{*}=n_{r}^{*}
$$

or there is $a \gamma>0$ such that

$$
e(n+1, r) \geqslant \gamma e(n, r)
$$

for all $n$ sufficiently large. Then for any compact $S$, not of finite rank, we have

$$
\lim _{n \rightarrow \infty} \frac{e(n, s)}{e(n, r)}=0 \text {. }
$$

Note that (5.48) or (5.49) holds in all of the examples mentioned in Section 1. The condition (5.49) tells us that the problem $\left(S, \Re H^{r}(\Omega)\right)$ cannot be "too easy", i.e., the error should decay no faster than geometrically.

On the other hand, we now show that a result like (5.50) cannot hold for all problems.

TheOREM 5.2. For any $\mu \in[0, \infty]$, there exists an $S$ for which

$$
\lim _{n \rightarrow \infty} \frac{e(n, s)}{e(n, r)}=\mu \text {, }
$$

and if $\mu=\infty$, the limit can go to infinity arbitrarily fast.

Proof. Setting $m=n_{s}^{*}-n_{r}^{*}$, we may use (5.46) and (5.47) to find

$$
\lim _{n \rightarrow \infty} \frac{e(n, s)}{e(n, r)}=\lim _{n \rightarrow \infty} \frac{r\left(n, \hat{S} \hat{E}, B \hat{H}^{s}(\Omega)\right)}{r\left(n+m, \hat{S}, B \hat{H}^{r}(\Omega)\right)} .
$$

Now let $X=\hat{H}^{s}(\Omega), Y=\hat{H}^{r}(\Omega)$, and $E=\hat{E}$ in the notation preceding Theorem 3.2.

Let $\hat{S}: Y \rightarrow Y$ be an injection. Let $Z=P_{r-1}(\Omega) \times \hat{H}^{r}(\Omega)$ with norm

$$
\left\|\left(\begin{array}{c}
\hat{p} \\
\hat{f}
\end{array}\right)\right\|^{2}:=\|\hat{p}\|_{r}^{2}+|\hat{f}|_{r}^{2} \quad \forall\left(\begin{array}{c}
\hat{p} \\
\hat{f}
\end{array}\right) \in Z .
$$

Finally, $S: H^{r}(\Omega) \rightarrow Z$ is given by

$$
S f:=\left(\begin{array}{c}
\hat{p} \\
\hat{S} \hat{f}
\end{array}\right)
$$

where $f=\hat{f}+\hat{p}$ as in (5.16).

By construction, $S$ is injective, so that (2.16) yields

$$
n_{r}^{*}=\operatorname{dim} P_{r-1}(\Omega)
$$


and

$$
n_{s}^{*}=\operatorname{dim} P_{s-1}(\Omega) \text {. }
$$

Hence $m>0$. Now finally choose $\hat{S}=A^{1 / 2}$, where $A$ is defined as in Theorem 3.2, with $\mu$ replaced by $\mu^{2}$. Then

$$
\lim _{n \rightarrow \infty} \frac{r\left(n, \hat{S} \hat{E}, B \hat{H}^{x}(\Omega)\right)}{r\left(n+m, \hat{S}, B \hat{H}^{r}(\Omega)\right)}=\left[\lim _{n \rightarrow \infty} \frac{\lambda_{n}\left(E^{*} A E\right)}{\lambda_{n+m}(A)}\right]^{1 / 2}=\mu .
$$

The theorem now follows from (5.51) and (5.56).

Hence, the penalty for increasing regularity may be arbitrarily great in the seminormed case.

6. Complexity Results. In this section, we translate our results on optimal error behavior into results on computational complexity. We show (roughly speaking) that both in the normed and seminormed cases, increasing regularity improves complexity; however, there are problems for which improvement means only that the complexity gets no worse.

The model of computation will be that specified by Chapter 5 of [6]. That is, if $H$ is a Hilbert space, evaluation of $\alpha f$ and $f+g(\alpha$ a scalar, $f, g \in H$ ) and evaluation of a linear functional on $H$ have finite complexity. We let $c$ denote the complexity of evaluating a linear functional; we assume that evaluation of $\alpha f$ and $f+g$ have unit complexity, in order to normalize the measure of complexity. We generally would expect $c \gg 1$.

We first consider the normed case. Let

$$
\operatorname{comp}(\varepsilon, r):=\inf _{\varphi} \operatorname{comp}(\varphi) .
$$

where $\operatorname{comp}(\varphi)$ denotes the complexity of the algorithm $\varphi$ for the problem $\left(S, B H^{r}(\Omega)\right)$ and the infimum is taken over all such algorithms $\varphi$ for which $e(\varphi) \leqslant \varepsilon$. Similarly,

$$
\operatorname{comp}(\varepsilon, s):=\inf _{\varphi} \operatorname{comp}(\varphi)
$$

where the infimum is now taken over all algorithms $\varphi$ for the problem $\left(\tilde{S}, B H^{s}(\Omega)\right)$ for which $e(\varphi) \leqslant \varepsilon$. Define the $\varepsilon$-cardinality numbers by

$$
m(\varepsilon, t):=\inf N(\varepsilon, t),
$$

where

$$
N(\varepsilon, t):=\left\{n \in \mathbf{Z}^{+}: r\left(n, B H^{t}(\Omega)\right) \leqslant \varepsilon\right\}
$$

for $t=r$ and $t=s$. (That is, $m(\varepsilon, t)$ is the smallest integer $n$ such that the $n$th minimal radius of information is at most $\varepsilon$ for data in the unit ball of $H^{t}(\Omega)$.) Then Theorem 3.4.2 and Lemma 5.2.2 of [6] yield

$$
\operatorname{comp}(\varepsilon, t)=\left(c+a_{t}\right) m(\varepsilon, t)-1,
$$

where $a_{t} \in[1,2]$.

We first discuss the behavior of the $\varepsilon$-cardinality numbers. 
THEOREM 6.1. Let $s>r$.

(i) For any $\varepsilon>0$, and any solution operator, $m(\varepsilon, s) \leqslant m(\varepsilon, r)$.

(ii) There exists a solution operator for which

$$
\lim _{\varepsilon \rightarrow 0} \frac{m(\varepsilon, s)}{m(\varepsilon, r)}=1 \text {. }
$$

Proof. (i) follows from the first statement in Theorem 4.2. To see (ii), let $y_{1}, y_{2}, \ldots$ be the orthonormal eigenfunctions of $E^{*} E$ corresponding to eigenvalues $\varepsilon_{1}^{2} \geqslant \varepsilon_{2}^{2} \geqslant$ $\cdots \geqslant 0$. Recall that $\varepsilon_{n} \sim c n^{-\mu}$ for $\mu=(s-r) / N$. Now define $S: H^{r}(\Omega) \rightarrow H^{r}(\Omega)$ by

$$
S E y_{n}=e^{1-n} E y_{n} .
$$

(Recall that $\left\{E y_{1}, E y_{2}, \ldots\right\}$ is complete in $H^{r}(\Omega)$.) Then $\lambda_{n}\left(S^{*} S\right)=e^{2(1-n)}$ and $\lambda_{n}\left((S E)^{*}(S E)\right) \sim\left[c n^{-\mu} e^{(1-n)}\right]^{2}$, i.e.,

$$
r\left(n, B H^{r}(\Omega)\right)=e^{-n} \text { and } r\left(n, B H^{s}(\Omega)\right) \sim c n^{-\mu} e^{-n} .
$$

We then have

$$
m(\varepsilon, r)=\ln \frac{1}{\varepsilon} \quad \text { and } \quad m(\varepsilon, s) \sim \ln \frac{1}{\varepsilon},
$$

completing the proof of the theorem.

We then have

THEOREM 6.2. Let $s>r$ in the normed case.

(i) For any solution operator,

$$
\operatorname{comp}(\varepsilon, s) \leqslant\left(\frac{c+a_{s}}{c+a_{r}}\right) \operatorname{comp}(\varepsilon, r)+\frac{a_{s}-a_{r}}{c+a_{r}} \quad \forall \varepsilon>0,
$$

so that, if $S$ is not of finite rank,

$$
\limsup _{\varepsilon \rightarrow 0} \frac{\operatorname{comp}(\varepsilon, s)}{\operatorname{comp}(\varepsilon, r)} \leqslant \frac{c+a_{s}}{c+a_{r}} .
$$

(ii) There exists a solution operator for which

$$
\lim _{\varepsilon \rightarrow 0} \frac{\operatorname{comp}(\varepsilon, s)}{\operatorname{comp}(\varepsilon, r)}=\frac{c+a_{s}}{c+a_{r}}
$$

Proof. Immediate from (6.5) and Theorem 6.1.

Since we generally expect $c \gg 1$, the first part of Theorem 6.2 tells us that

$$
\limsup _{\varepsilon \rightarrow 0} \frac{\operatorname{comp}(\varepsilon, s)}{\operatorname{comp}(\varepsilon, r)} \leqslant \frac{c+2}{c+1} \approx 1,
$$

while the second part of the theorem tells us that there is a problem for which

$$
\lim _{\varepsilon \rightarrow 0} \frac{\operatorname{comp}(\varepsilon, s)}{\operatorname{comp}(\varepsilon, r)} \geqslant \frac{c+1}{c+2} \approx 1 .
$$

We may roughly paraphrase (6.9) by saying that increasing regularity improves complexity; (6.10) tells us that there are problems for which "improvement" means only that the behavior of the complexity does not get worse. 
We now consider the seminormed case. Let

$$
\operatorname{comp}(\varepsilon, r)=\inf _{\varphi} \operatorname{comp}(\varphi),
$$

where $\operatorname{comp}(\varphi)$ is the complexity of the algorithm $\varphi$ for the problem $\left(S, \circlearrowleft B H^{r}(\Omega)\right)$ and the infimum is taken over all such algorithms $\varphi$ for the problem $\left(S, 9 h H^{r}(\Omega)\right)$ for which $e(\varphi) \leqslant \varepsilon$. Similarly,

$$
\operatorname{comp}(\varepsilon, s):=\inf _{\varphi} \operatorname{comp}(\varphi),
$$

with the infimum now being taken over all algorithms $\varphi$ for the problem $\left(\tilde{S}, \mathscr{B} H^{s}(\Omega)\right)$ for which $e(\varphi) \leqslant \varepsilon$. We now define the $\varepsilon$-cardinality numbers by

$$
m(\varepsilon, t):=\inf N(\varepsilon, t) \text {, }
$$

where now

$$
N(\varepsilon, t):=\left\{n \in \mathbf{Z}^{+}: r\left(n, \cup B H^{t}(\Omega)\right)<\varepsilon\right\}
$$

for $t=r$ and $t=s$. Then (as in the normed case) there is an $a_{t} \in[1,2]$ for which

$$
\operatorname{comp}(\varepsilon, t)=\left(c+a_{t}\right) m(\varepsilon, t)-1
$$

for $t=r$ and $t=s$.

We first discuss the behavior of the $\varepsilon$-cardinality numbers.

THEOREM 6.3. (i) For any solution operator, there exists $\varepsilon_{0}>0$ such that

$$
m(\varepsilon, s) \leqslant m(\varepsilon, r)+n_{s}^{*}-n_{r}^{*} \quad \forall \varepsilon \in\left(0, \varepsilon_{0}\right],
$$

and so if $S$ is not of finite rank.

$$
\limsup _{\varepsilon \rightarrow 0} \frac{m(\varepsilon, s)}{m(\varepsilon, r)} \leqslant 1 .
$$

(ii) There exists a solution operator for which

$$
\lim _{\varepsilon \rightarrow 0} \frac{m(\varepsilon, s)}{m(\varepsilon, r)}=1 .
$$

Proof. (i) By Theorem 4.2, $\lim _{r \rightarrow \infty}\left[r\left(n, B \hat{H}^{s}(\Omega)\right) / r\left(n, B \hat{H}^{r}(\Omega)\right)\right]=0$. Hence there is an $n_{0} \in \mathbf{Z}^{+}$such that

$$
r\left(n, B \hat{H}^{s}(\Omega)\right) \leqslant r\left(n, B \hat{H}^{r}(\Omega)\right) \quad \forall n \geqslant n_{0},
$$

so that Lemma 5.3 yields

$$
r\left(n+n_{s}^{*}, \mathscr{B} H^{s}(\Omega)\right) \leqslant r\left(n+n_{r}^{*}, \mathscr{B} H^{r}(\Omega)\right) \quad \forall n \geqslant n_{0} .
$$

Let

$$
\varepsilon_{0}:=r\left(n_{0}+n_{r}^{*}, 9 B H^{r}(\Omega)\right) .
$$

To prove (6.16), let $\varepsilon \in\left(0, \varepsilon_{0}\right]$. If $N(\varepsilon, r)$ is empty, the right-hand side of $(6.16)$ is infinite, so that (6.16) is trivial. So, let $n \in N(\varepsilon, r)$. Since

$$
r\left(n, \mathscr{B} H^{r}(\Omega)\right) \leqslant \varepsilon \leqslant \varepsilon_{0}=r\left(n_{0}+n_{r}^{*}, \mathscr{B}_{B} H^{r}(\Omega)\right)
$$

and $r\left(\cdot, \mathscr{B} H^{r}(\Omega)\right)$ is nonincreasing, we have $n \geqslant n_{0}+n_{r}^{*}$, i.e.,

$$
n-n_{r}^{*} \geqslant n_{0} \text {. }
$$


Using (6.19), and replacing " $n$ " by " $n-n_{r}^{*}$ " in (6.18), we have

$$
r\left(n+n_{s}^{*}-n_{r}^{*}, \mathscr{B} H^{s}(\Omega)\right) \leqslant r\left(n, \mathscr{B} H^{r}(\Omega)\right) \leqslant \varepsilon,
$$

so that $n+n_{s}^{*}-n_{r}^{*} \in N(\varepsilon, s)$; so,

$$
m(\varepsilon, s)=\inf N(\varepsilon, s) \leqslant n+n_{s}^{*}-n_{r}^{*} .
$$

Since $n \in N(\varepsilon, r)$ is arbitrary, (6.16) follows.

To prove (6.17), let $S$ not be of finite rank. Then $\lim _{\varepsilon \rightarrow 0} m(\varepsilon, r)=\infty$, so that (6.16) yields (6.17).

To prove (ii), let $y_{1}, y_{2}, \ldots$ be the orthonormal eigenfunctions of $\hat{E}^{*} \hat{E}$ corresponding to eigenvalues $\varepsilon_{1}^{2} \geqslant \varepsilon_{2}^{2} \geqslant \cdots \geqslant 0$. Again $\varepsilon_{n} \sim c n^{-\mu}$ for $\mu=(s-r) / N$. Let $S$ : $H^{r}(\Omega) \rightarrow H^{r}(\Omega)$ be such that

$$
\hat{S} \hat{E} y_{n}=e^{1-n} \hat{E} y_{n},
$$

and let $S$ restricted to $P_{r-1}(\Omega)$ be the zero operator. Then as in the proof of Theorem 6.1 we have

$$
r\left(n, \Re H^{r}(\Omega)\right)=e^{-n} \text { and } r\left(n, \Re H^{s}(\Omega)\right) \sim c n^{-\mu} e^{-n},
$$

so that

$$
m(\varepsilon, r)=\ln \frac{1}{\varepsilon} \quad \text { and } m(\varepsilon, s) \sim \ln \frac{1}{\varepsilon},
$$

completing the proof of the theorem.

Remark 6.1. Note that Theorem 6.3 gives an asymptotic result, i.e., one for all sufficiently small $\varepsilon$. One can also prove the nonasymptotic result

$$
m(\varepsilon\|\hat{E}\|, s) \leqslant m(\varepsilon, r)+n_{s}^{*}-n_{r}^{*} \quad \forall \varepsilon>0 .
$$

When $\|\hat{E}\| \leqslant 1$, (6.23) implies that (6.16) holds for all $\varepsilon>0$. However, it is possible to choose $\Omega$ so that $\|\hat{E}\|>1$. (For example, let $r=0, s=1, N=1$; then set $\Omega=(-a, a)$ with $a>\sqrt{3}$.) In this case, (6.23) does not imply that (6.16) holds for all $\varepsilon>0$. In fact, when $\|\hat{E}\|>1$, one can construct a solution operator for which (6.16) does not hold for all $\varepsilon>0$.

To see this, let $S: H^{r}(\Omega) \rightarrow H^{r}(\Omega)$ be given by

$$
\hat{S} \hat{E} y_{n}=\sigma_{n} \hat{E} y_{n} \text {, }
$$

with $\sigma_{1} \geqslant \sigma_{2} \geqslant \cdots \geqslant 0$ and $y_{n}$ as in the proof above, and define $S$ to be zero on $P_{r-1}(\Omega)$. Then

$$
r\left(n, B \hat{H}^{r}(\Omega)\right)=\sigma_{n+1} \quad \text { and } \quad r\left(n, B \hat{H}^{s}(\Omega)\right)=\sigma_{n+1} \varepsilon_{n+1},
$$

so that $\|\hat{E}\|>1$ yields

$$
r\left(0, B \hat{H}^{s}(\Omega)\right)=\sigma_{1} \varepsilon_{1}=\sigma_{1}\|\hat{E}\|=\|\hat{E}\| r\left(0, B \hat{H}^{r}(\Omega)\right)>r\left(0, B \hat{H}^{r}(\Omega)\right),
$$

i.e., Lemma 5.3 yields

$$
r\left(n_{s}^{*}, \mathscr{B} H^{s}(\Omega)\right)>r\left(n_{r}^{*}, \mathscr{B} H^{r}(\Omega)\right) .
$$

Now let

$$
\varepsilon:=r\left(n_{r}^{*}, \Re H^{r}(\Omega)\right)
$$

Then

$$
m(\varepsilon, r)=n_{r}^{*},
$$


while (6.24) yields $r\left(n_{s}^{*},\left(B H^{s}(\Omega)\right)>\varepsilon\right.$, so that

$$
m(\varepsilon, s)>n_{s}^{*} .
$$

From (6.25) and (6.26), we find

$$
m(\varepsilon, s)>m(\varepsilon, r)+n_{s}^{*}-n_{r}^{*} .
$$

So, (6.16) cannot hold for all $\varepsilon>0$.

We may then use (6.15) and Theorem 6.3 to prove

THEOREM 6.4. Let $s>r$ in the seminormed case.

(i) For any solution operator, there exists $\varepsilon_{0}>0$ such that

$$
\begin{aligned}
\operatorname{comp}(\varepsilon, s) \leqslant & \left(\frac{c+a_{s}}{c+a_{r}}\right) \operatorname{comp}(\varepsilon, r)+\left(c+a_{s}\right)\left(n_{s}^{*}-n_{r}^{*}\right) \\
& +\frac{a_{s}-a_{r}}{c+a_{r}}, \quad \forall \varepsilon \in\left(0, \varepsilon_{0}\right],
\end{aligned}
$$

so that for $S$ not of finite rank,

$$
\limsup _{r \rightarrow 0} \frac{\operatorname{comp}(\varepsilon, s)}{\operatorname{comp}(\varepsilon, r)} \leqslant \frac{c+a_{s}}{c+a_{r}} .
$$

(ii) There exists a solution operator for which

$$
\lim _{\varepsilon \rightarrow 0} \frac{\operatorname{comp}(\varepsilon, s)}{\operatorname{comp}(\varepsilon, r)}=\frac{c+a_{1}}{c+a_{r}}
$$

Since we generally expect $c \gg 1$, we would expect $\left(c+a_{s}\right) /\left(c+a_{r}\right) \approx 1$. Hence this theorem tells us that increasing regularity improves complexity, although there are problems for which the improvement means that only the complexity gets no worse.

7. Open Questions. In this paper, we have examined the role of regularity in determining complexity. Here, we consider some open problems in this area.

We first consider the normed case. We saw that

$$
\lim _{n \rightarrow x} e(n, s) / e(n, r)=0 \text { when } r<s .
$$

Is there any way of measuring how fast the ratio tends to zero, given (say) $r, s$, and some knowledge of the solution operator $S$ ? There appears to be no way of extending the proof in this paper (which uses a compactness argument) to find such a rate.

It would also be reasonable to consider problems defined over the Sobolev space $W^{r . p}(\Omega)$. In this case, it is easy to see that $d(n, s) \leqslant d(n, r)$ for all $n$ when $s>r$. where $d(n, t)$ is the $n$th minimal diameter of information for data in $B W^{t . p}(\Omega)$ (see [6, p. 11]). Is it still true that $\lim _{n \rightarrow \infty} e(n, s) / e(n, r)=0$ ? The proof of such a statement would follow from a theorem on ratios of $n$-widths, similar in flavor to Theorem 3.1. Such a theorem ("increasing compactness speeds up the decay of $n$-widths") is plausible, but its proof could not use the eigenspace techniques of Section 3.

We now consider the seminormed case. Although we know that there exist problems for which increasing regularity is harmful (in the sense of Theorem 5.2), we 
know of no naturally-occurring problem for which this is the case. Is there a noncontrived problem for which increasing regularity worsens the asymptotic behavior of the optimal error?

It is also of interest to find classes of problems for which increasing regularity (again, in the seminormed sense) improves the asymptotic behavior of the optimal error. For example, if we look at problems defined over $9 B H_{0}^{r}(\Omega)$, the Friedrichs inequality allows us to use the results in Section 4, so that

$$
\lim _{n \rightarrow \infty} e(n, s) / e(n, r)=0 \text {. }
$$

Corollary 5.1 gives other conditions which are sufficient to yield this result. What are necessary and sufficient conditions for increasing seminorm regularity to improve the asymptotic behavior of the optimal error in the seminormed case?

We next note that the strongest statement that one can make is (roughly) that increasing regularity does not make the complexity worse; this is because there exist problems for which increasing regularity leaves the complexity unchanged. On the other hand, for many naturally-occurring problems, we have

$$
\lim _{r \rightarrow 0} \frac{\operatorname{comp}(\varepsilon, s)}{\operatorname{comp}(\varepsilon, r)}=0 \text { whenever } r<s .
$$

It would be useful to characterize the problems for which this holds, while an even more ambitious task would be characterizing the problems for which the complexity ratio goes to zero as a given function of $\varepsilon$.

Finally, we point out that this paper only deals with linear solution operators. Does increased regularity lower complexity when the solution operator is nonlinear?

Acknowledgements. I would like to thank Professors T. I. Seidman (University of Maryland Baltimore County), J. F. Traub (Columbia University), and $\mathbf{H}$. Woźniakowski (University of Warsaw) for their comments and suggestions concerning the results reported in this paper.

\footnotetext{
Division of Science and Mathematics

Fordham University

College at Lincoln Center

New York, New York 10023

Department of Computer Science

Columbia University

New York, New York 10027
}

1. I. BABUŠKA \& A. K. AzIz, "Survey lectures on the mathematical foundations of the finite element method," in The Mathematical Foundations of Finite Element Method with Applications to Partial Differential Equations (A. K. Aziz, ed.), Academic Press, New York, 1972, pp. 3-359.

2. P. (i. Ciariet, The Finite Element Method for Elliptic Problems, Studies in Mathematics and Its Applications, Vol. 4 (J. L. Lions et al., eds.), North-Holland, Amsterdam, 1978.

3. A. Friedman, Foundations of Modern Analysis, Holt, Rinehart, and Winston, New York, 1970.

4. J. NeČAs, Les Méthodes Directes en Théorie des Equations Elliptiques, Masson, Paris, 1967.

5. M. Schfchter, Principles of Functional Analysis, Academic Press, New York, 1971.

6. J. F. Traub \& H. WoŹniakowski, A General Theory of Optimal Algorithms, Academic Press, New York, 1980).

7. A. (j. Werschulz, "Optimal-error properties of finite-element methods for second-order elliptic Dirichlet problems," Math. Comp., v. 38, 1982, pp. 401-413. 